\title{
Macrophage migration inhibitory factor knockdown inhibit viability and induce apoptosis of PVM/Ms
}

\author{
WENJING ZHANG, JIAN ZHENG, JUAN MENG, LINGLING NENG, XIAOHUA CHEN and ZHAOBING QIN \\ Department of Otology, The First Affiliated Hospital of Zhengzhou University, Zhengzhou, Henan 450000, P.R. China
}

Received November 18, 2016; Accepted July 3, 2017

DOI: $10.3892 / \mathrm{mmr} .2017 .7684$

\begin{abstract}
Previous studies have suggested that macrophage migration inhibitory factor (MIF) serves an important role in hearing function; however, the underlying mechanism remains unclear. In the present study, perivascular-resident macrophage-like melanocytes (PVM/Ms) from the stria vascularis of the lateral cochlear wall in young and aged mice were isolated. The mRNA and protein expression levels of MIF were determined using reverse transcription-quantitative polymerase chain reaction analysis, and western blotting, respectively. MIF expression was knocked down in vitro and in vivo using small interfering RNA. Cell viability was determined using an MTT assay and cell apoptosis was determined using flow cytometry analysis. The hearing ability was assessed through the auditory brain stem response in vivo. The results of the current study demonstrated that the expression of MIF was significantly downregulated in aged mice compared with in young mice. Furthermore, the viability of $\mathrm{PVM} / \mathrm{Ms}$ in aged mice was significantly decreased and the number of apoptotic PVM/Ms was significantly increased compared with that in young mice. Further studies demonstrated that the MIF knockdown accentuated hearing loss in young mice as compared with the scramble control group. In addition, the MIF knockdown in PVM/Ms significantly inhibited cell viability and lead to a significant increase in the apoptotic cell number as compared with the control group. In summary, these results revealed that the MIF knockdown significantly accentuates hearing loss in young mice in vivo, and significantly inhibits the viability and induces the apoptosis of PVM/Ms in vitro. Thus, the results of the present study may provide a novel potential therapeutic approach and prevention method for presbycusis.
\end{abstract}

Correspondence to: Dr Zhaobing Qin, Department of Otology, The First Affiliated Hospital of Zhengzhou University, 1 Jianshe Road, Zhengzhou, Henan 450000, P.R. China

E-mail: qin_zb@sohu.com

Key words: macrophage migration inhibitory factor, perivascularresident macrophage-like melanocytes, presbycusis

\section{Introduction}

Presbycusis, also known as age-related hearing loss (AHL), is a common neurodegenerative disorder and characterized by gradual, progressive sensorineural hearing loss (1). Presbycusis affects $40 \%$ of patients between 65 and 66 years old and $66.8 \%$ of patients between 73 and 74 years old (2). Presbycusis can result in the speech processing deficits, the slowed central processing of acoustic stimuli, the impaired sound localization, social isolation, depression, and even cognitive impairment $(1,3-5)$. Thus, have a better understanding of the pathogenesis of presbycusis is of great importance to the therapy and prevention of presbycusis.

It is well known that the intrastrial fluid-blood barrier could separate the stria vascularis from peripheral circulation. Especially, the integrity of the barrier is vital important for maintaining inner ear homeostasis and sustaining the endocochlear potential, which is an essential driving force for hearing function (6-9). A previous study indicated that the intrastrial fluid-blood barrier comprises a large number of perivascular-resident macrophage-like melanocytes (PVM/Ms) $(10,11)$. Further study verified that absence of PVM/Ms increases the permeability of the intrastrial fluid-blood barrier to both low and high-molecular-weight tracers (12). Totally, these investigations may indicate the important role of PVM/Ms in hearing function.

Macrophage migration inhibitory factor (MIF) is an essential factor in axis and neural development. MIF is reported to be strongly expressed in the inner ear and plays an important role in inner ear of mice (13). Additionally, MIF was proved to play an important role in cell viability signal transmission, as well as in cell growth $(14,15)$. Thus, it raises the possibility that MIF may via regulating the growth of PVM/Ms and exert important roles in hearing function.

To validate our speculation, $\mathrm{PVM} / \mathrm{Ms}$ from stria vascularis of lateral wall of cochlear in young and aged mice were isolated. We found the expression of MIF was significantly downregulated in aged mice. The viability of PVM/Ms in aged mice was significantly decreased, and the apoptotic PVM/Ms number in aged mice was markedly increased. Further studies showed that MIF knockdown in young mice had more serious hearing loss as compared to the control. MIF knockdown in PVM/Ms also revealed the significant inhibited cell viability, the markedly induced cell apoptosis as compared to the control. Totally, our results revealed that MIF knockdown in vivo significantly 
accentuated hearing loss in young mice, and MIF knockdown in vitro significantly inhibited viability and induced apoptosis of PVM/Ms. Our study may provide a potential therapy and prevention method for presbycusis.

\section{Materials and methods}

Animals. All experiments were approved by the ethical committee of the First Affiliated Hospital of Zhengzhou University. C57BL/6J mice, which are known for their rapid development of hearing loss, particularly in the high-frequency range, by one year of age $(16,17)$. Mice were purchased from the Chinese Academy of Medical Sciences (Beijing, China). In our study, mice aged 2 months and 10 months were termed as the young group and aged group, respectively ( $\mathrm{n}=20$ in each group). The mice were housed 2 or 3 per cage and had free access to food and water, with the suitable temperature and humidity conditions, along with a 12/12 h light/dark cycle.

PVM/Ms isolation and culture. PVM/Ms were isolated from mice aged 2 months and 10 months, respectively, and were cultured as previously described $(12,18)$. The stria vascularis of lateral wall of cochlear was firstly isolated. To producePVM/Ms, the minced stria vascularis was cultured on collagen-coated dishes in medium 254 (Invitrogen Life Technologies, Carlsbad, CA, USA), which containing 10\% FBS, $1 \%$ human melanocyte growth factor and $0.5 \%$ gentamicin/amphotericin. The minced stria vascularis was incubated at $37^{\circ} \mathrm{C}$ in $5 \% \mathrm{CO}_{2}$ with the medium changed every 3 days. Cell clones formed and melted at approximately 10 days. The cells were detached from the cell colony with a solution of trypsin-EDTA and then were purified. For purification of PVM/M cells, antibody F4/80 or GST were added to the medium. Cells were incubated with the antibodies for $10 \mathrm{~min}$ at $4^{\circ} \mathrm{C}$, and then for isolation, and were grown in medium 254 containing $1 \%$ human melanocyte growth factor.

siRNA transfection. MIF silencing in vitro was performed on passage-3 PVM/Ms seeded in 24-well plates as described previously (12). The PVM/Ms ( $1 \times 10^{5}$ cells/well) were transfected with si-MIF and scrambled siRNA (Applied Biosystems) according to the manufacturer's guidelines. After 4 days, the transfection efficiency was determined by western blotting and cells were used for cell viability and apoptosis assay.

The in vivo siRNA transfection was performed as described previously $(12,19)$. Briefly, animals were anesthetized, and a 30-G needle was used to make a single puncture in the anterior-inferior quadrant of the tympanic membrane to allow exit of air from the middle ear during drug injection. MIF was silenced with a $5-\mu \mathrm{l}$ solution of siRNA $(20 \mathrm{ng} / \mu \mathrm{l})$ injected through the posterior-inferior quadrant. The middle ear was filled completely with the solution for 5 days $(n=3$ mice per group). Scrambled siRNA of the same concentration was given to control group ( $\mathrm{n}=3$ mice per group). At 8 days after the in vivo siRNA transfection, the transfection efficiency was determined by western blotting and used for hearing tests.

Cell viability assay. Cells $\left(4 \times 10^{3}\right.$ cells/well) were seeded into the 96-well plate overnight. MTT was added, and then incubated for $4 \mathrm{~h}$. The formazan crystals formed from MTT by the living cells were dissolved in $150 \mu 1$ dimethyl sulfoxide (DMSO), and then detected using a spectrophotometer at an absorption wavelength of $490 \mathrm{~nm}$.

Flow cytometry analysis for apoptosis. The Annexin V-FITC/PI Apoptosis Detection kit (Roche Diagnostics, Rotkreuz, Switzerland) was used to detect cell apoptosis. The cells were washed with cold PBS and resuspended. Staining was performed according to the producer's manual. Flow cytometry (BD Biosciences, Franklin Lakes, NJ, USA) was performed immediately.

Hearing tests. The auditory brain stem response (ABR) test has been described previously (20). Briefly, hearing was measured with a click stimulus and at 4, 8, 16 and $32 \mathrm{kHz}$ using tone pips ( $3 \mathrm{msec}$ duration). Stimuli were presented to both ears simultaneously at decreasing intensities ( $5 \mathrm{~dB}$ steps) in a recording system. For each animal for each stimulus, the threshold response was recorded.

Reverse transcription-quantitative polymerase chain reaction $(q R T-P C R)$. Total RNA from purified PVM/Ms was extracted separately with a RNeasy kit (Qiagen, Valencia, CA, USA) according to the manufacturer's suggestions. One microgram of total RNA was reverse-transcribed using a SuperScript II (Invitrogen Life Technologies) following the manufacturer's instructions. RT-qPCR was performed using a SYBR-Green II RT-qPCR kit (Toyobo Co., Ltd., Osaka, Japan) and a Prism 7300 Sequence Detection System (Applied Biosystems, Foster City, CA, USA). GAPDH was used as the internal control.

Western blot analysis. Total protein was isolated from purified $\mathrm{PVM} / \mathrm{Ms}$ and the protein concentration was determined. SDS-PAGE was used to separate the proteins and then the protein was transferred onto a nitrocellulose membrane (Millipore Corp., Boston, MA, USA). The membrane was then blocked and incubated with primary antibody. Protein bands were detected by incubation with horseradish peroxidase-conjugated secondary antibody and visualized with chemiluminescence reagent (Pierce Chemical Co., Rockford, IL, USA).

Statistical analysis. Data are presented as mean \pm SEM. The unpaired Student's t-test using Graph Pad Prism 5.0 (Hearne Scientific Software, Chicago, IL, USA) was used to perform the statistical analysis. $\mathrm{P}<0.05$ was considered to indicate a statistically significant difference.

\section{Results}

MIF was downregulated in PVM/Ms from stria vascularis of lateral wall of cochlear in aged mice. $\mathrm{PVM} / \mathrm{Ms}$ from stria vascularis of lateral wall of cochlear in young and aged mice were isolated. Firstly, the macrophage and melanocyte marker proteins, F4/80 and GST (21), were determined by qRT-PCR. As shown in Fig. 1A and B, the mRNA expression levels of F4/80and GST were significantly downregulated in PVM/Ms from aged mice than that in the young mice. The mRNA expression level of MIF was also significantly downregulated in PVM/Ms from aged mice than that in the young mice 
A

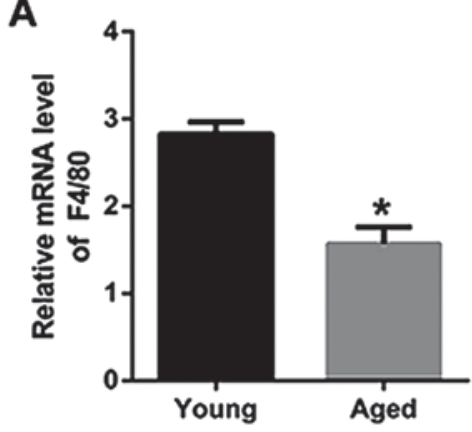

B

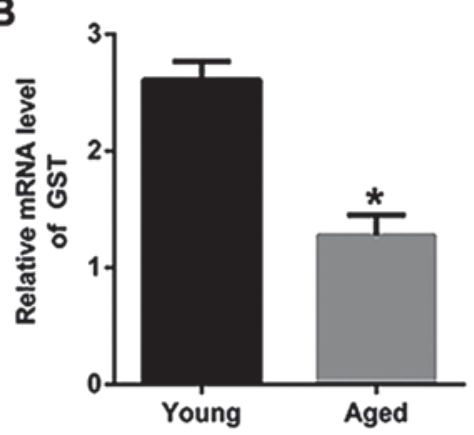

C

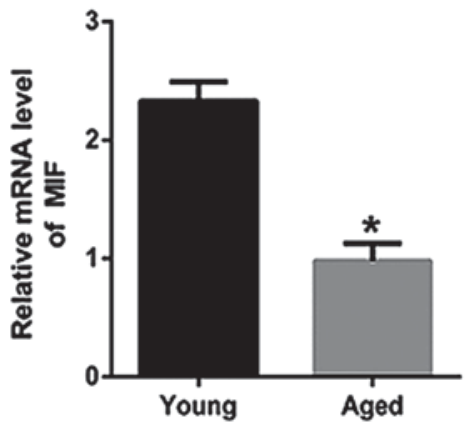

D

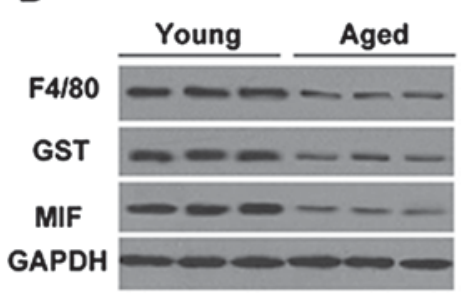

E

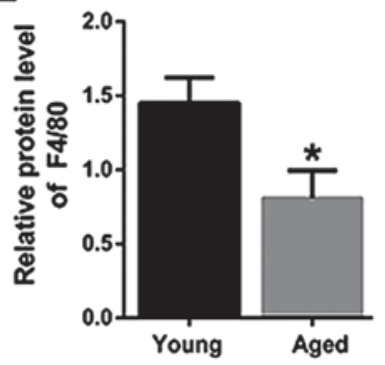

F

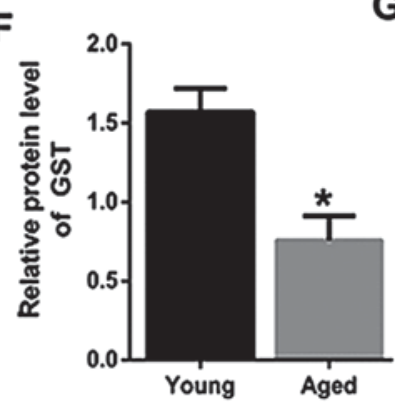

G

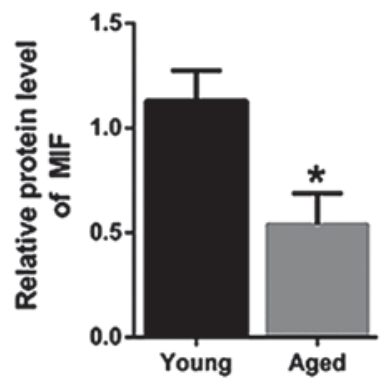

Figure 1. MIF was downregulated in PVM/Ms from stria vascularis of lateral wall of cochlear in aged mice. The mRNA expression level of (A) F4/80, (B) GST, and (C) MIF in PVM/Ms from aged mice and young mice (n=20 in each group). Purified PVM/Ms were isolated from mice and cultured, then for RT-qPCR analysis. (D) Protein bands of F4/80, GST and MIF in PVM/Ms from aged mice and young mice ( $\mathrm{n}=20$ in each group); The quantitative protein expression level of (E) F4/80, (F) GST and (G) MIF in PVM/Ms from aged mice and young mice (n=20 in each group). Purified PVM/Ms were isolated from mice and cultured, then for western blot analysis. ${ }^{*} \mathrm{P}<0.5$ vs. the young age group. RT-qPCR, reverse transcription-quantitative polymerase chain reaction; PVM/Ms, perivascular-resident macrophage-like melanocytes; MIF, migration inhibitory factor.

(Fig. 1C). Consistently, the decreased protein levels of F4/80, GST and MIF were also shown in aged mice as compared to that in the young mice (Fig. 1D-G). The significant reduction in F4/80 and GST indicated the decrease of PVM/Ms in aged mice, revealing the vital role of PVM/Ms in aged mice. More importantly, the reduction of MIF in aged mice was found, which may suggest the potential role of MIF in PVM/Ms growth.

The viability of PVM/Ms was decreased and the apoptotic $P V M / M$ s number was markedly increased in aged mice. The viability and apoptosis of PVM/Ms from young and aged mice were compared. The results revealed that the viability of PVM/Ms from aged mice was significantly decreased as compared to that from the aged mice (Fig. 2A). Inversely, the apoptosis of PVM/Ms from aged mice was significantly increased as compared to that from the aged mice (Fig. 2B). The cell population in G0/G1 phase of PVM/Ms from aged mice was significantly decreased as compared to that from young mice; whereas the cell population in $\mathrm{S}$ and $\mathrm{G} 2 / \mathrm{M}$ phases of PVM/Ms from aged mice was significantly increased as compared to that from young mice (Fig. 2C).

MIF knockdown in young mice leads to hearing loss. Considering the decreased cell viability of PVM/Ms and decreased protein level of MIF in aged mice, we knocked down MIF in young mice to explore the important role of MIF. MIF was knocked down in young mice and the in vivo transfection efficiency was determined by the western blotting (Fig. 3A). Through the ABR test, the results showed that the average threshold of the MIF knockdown group was maintained at a significant lower level than the control group, indicating the significant hearing loss in young mice with MIF knockdown (Fig. 3B).

MIF knockdown inhibited the viability and induced the apoptosis of $\mathrm{PVM} / \mathrm{Ms}$ from young mice. To further explore the underlying mechanism, PVM/Ms were isolated from young mice. MIF was then knocked down and transfected into PVM/Ms, the viability and apoptosis were determined respectively. The results revealed that MIF knockdown significantly inhibited the viability of PVM/Ms as compared to the control (Fig. 4A). Inversely, MIF knockdown significantly induced the apoptosis of PVM/Ms as compared to the control (Fig. 4B). The cell population in G0/G1 phase of $\mathrm{PVM} / \mathrm{Ms}$ with MIF knockdown was significantly decreased as compared to the control; whereas the cell population in $\mathrm{S}$ and G2/M phases of PVM/Ms with MIF knockdown was significantly increased as compared to the control (Fig. 4C). Totally, we found that the viability and apoptosis of PVM/Ms that transfected with si-MIF was comparable to that in the aged mice (Fig. 2).

\section{Discussion}

In present study, we firstly found the significant reduction in F4/80 and GST in aged mice. Since F4/80 and GST are the marker proteins of macrophage and melanocyte (21), to some extent, the results indicated the decrease of PVM/Ms in aged mice. We also found that the viability of PVM/Ms was 


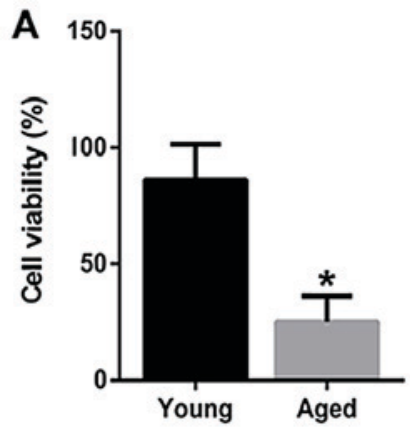

B
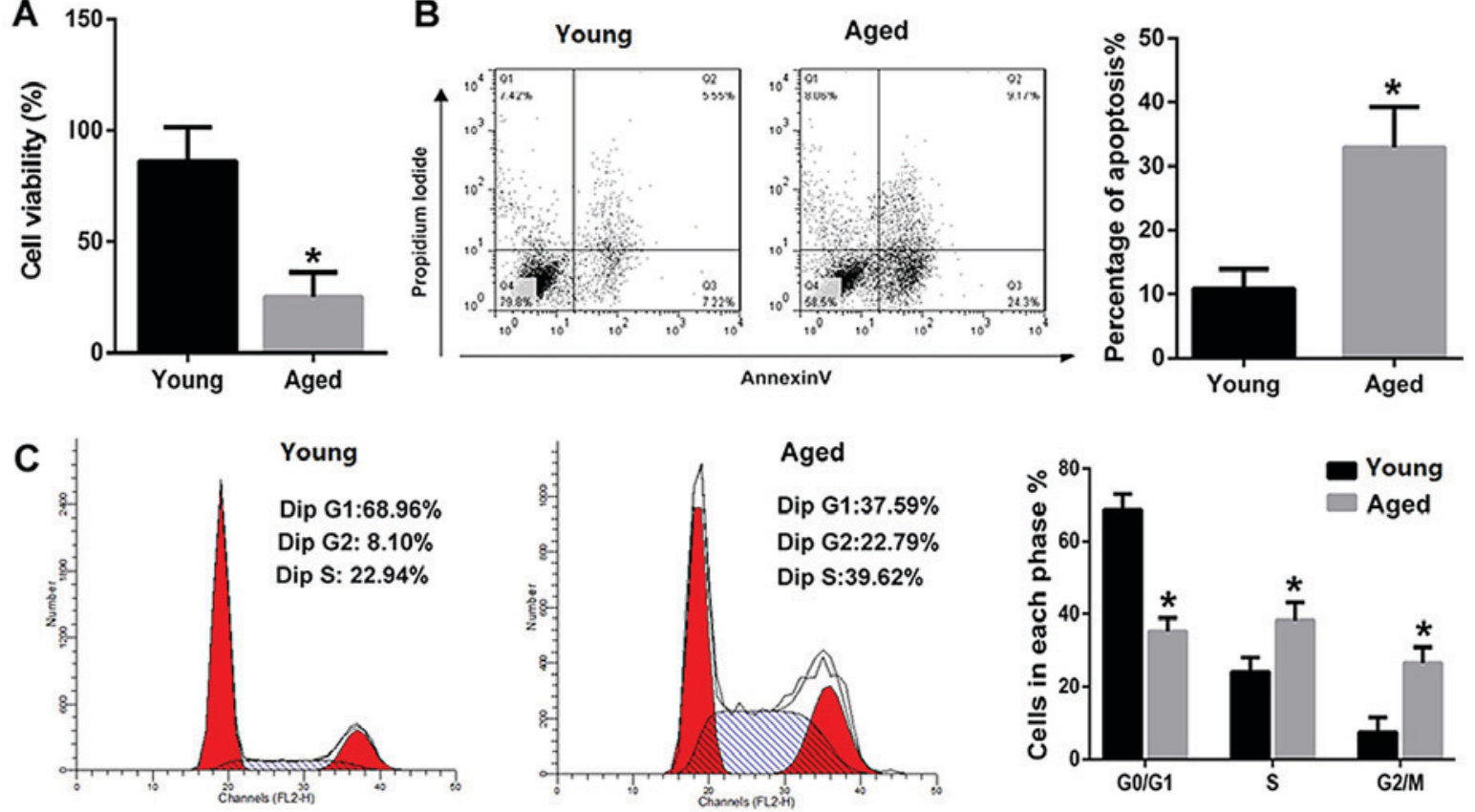

Figure 2. The viability of PVM/Ms was decreased and the apoptotic PVM/Ms number was markedly increased in aged mice. (A) The viability of PVM/Ms from aged mice was significantly decreased as compared to that from the aged mice ( $\mathrm{n}=20$ in each group). (B) The apoptosis of PVM/Ms from aged mice was significantly increased as compared to that from the aged mice ( $\mathrm{n}=20$ in each group). (C) The cell population in G0/G1 phase of PVM/Ms from aged mice was significantly decreased and the cell population in $\mathrm{S}$ and $\mathrm{G} 2 / \mathrm{M}$ phases of $\mathrm{PVM} / \mathrm{Ms}$ from aged mice was significantly increased as compared to that from young mice ( $\mathrm{n}=20$ in each group). Purified PVM/Ms were isolated from mice and cultured, then for the cell viability and apoptosis assay. ${ }^{*} \mathrm{P}<0.5$ vs. the young age group. PVM/Ms, perivascular-resident macrophage-like melanocytes.
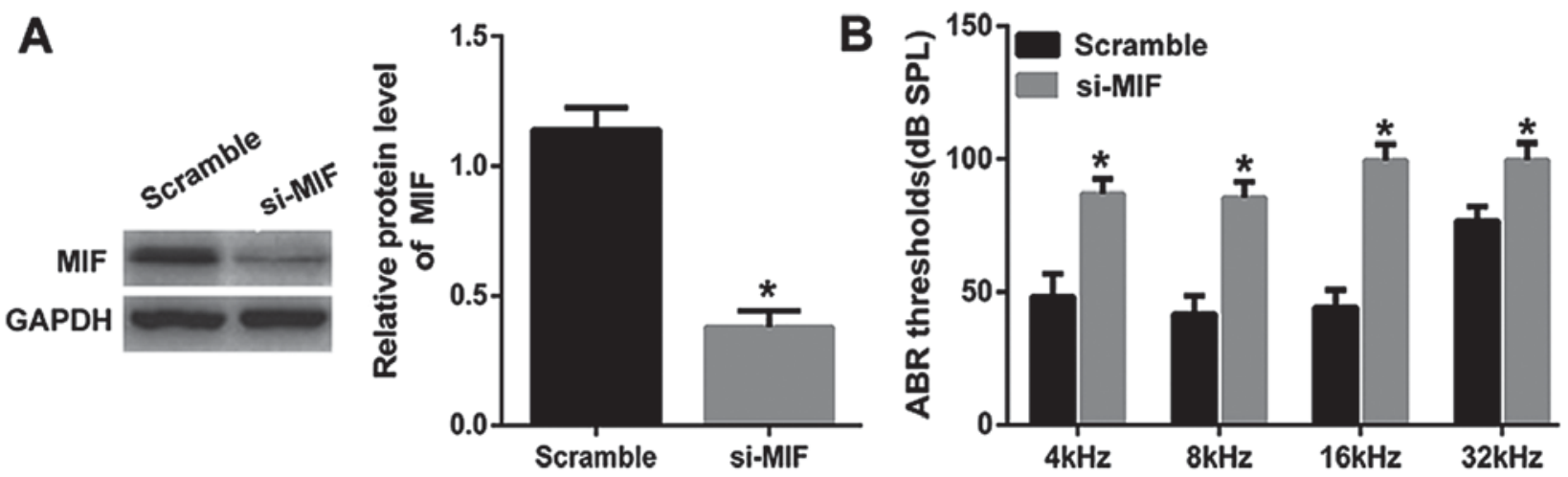

Figure 3. MIF knockdown in young mice leads to hearing loss (A) MIF was knocked down in young mice and the transfection efficiency was determined by the western blotting. (B) The average threshold of the MIF knockdown group was maintained at a significant lower level than the control group in the ABR test. The in vivo siRNA transfection was performed in young mice as described previously $(12,19)$. MIF was silenced with a $5-\mu 1$ solution of siRNA $(20 \mathrm{ng} / \mu \mathrm{l})$ injected through the posterior-inferior quadrant $(\mathrm{n}=3$ mice per group). Scrambled siRNA of the same concentration was given to control group ( $\mathrm{n}=3$ mice per group). At 8 days after the in vivo siRNA transfection, the transfection efficiency was determined and used for ABR tests. "P<0.5 vs. the Scramble group. MIF, migration inhibitory factor; ABR, auditory brain stem response.

decreased and the apoptotic PVM/Ms number was markedly increased in aged mice that that in young mice. Besides, the cell population in G0/G1 phase of PVM/Ms from aged mice was significantly decreased and the cell population in $\mathrm{S}$ and $\mathrm{G} 2 / \mathrm{M}$ phases of PVM/Ms from aged mice was significantly increased as compared to that from young mice. The progression of cell cycle was arrested at $\mathrm{S}$ and $\mathrm{G} 2 / \mathrm{M}$ phases as compared to that from young mice. To some extent, the results were consistent with the previous results demonstrating that $\mathrm{PVM} / \mathrm{Ms}$ are essential for maintaining cochlear vascular architecture and stability (22). Altogether, these investigations indicated the important role of PVM/Ms on hearing function. A previous study has suggested than the PVM/Ms are in close contact with vessels through cytoplasmic processes and have an important role in maintaining the integrity of the intrastrial fluid-blood barrier and hearing function. Besides, they also showed that absence of PVM/Ms increases the permeability of the intrastrial fluid-blood barrier to both low and high-molecular-weight tracers. Besides, in the PVM/M-depleted animals, substantial drop in endocochlear potential with accompanying hearing loss was found (12).

Based on these results, it seems that factors that affect $\mathrm{PVM} / \mathrm{Ms}$ growth were also associated with the hearing 

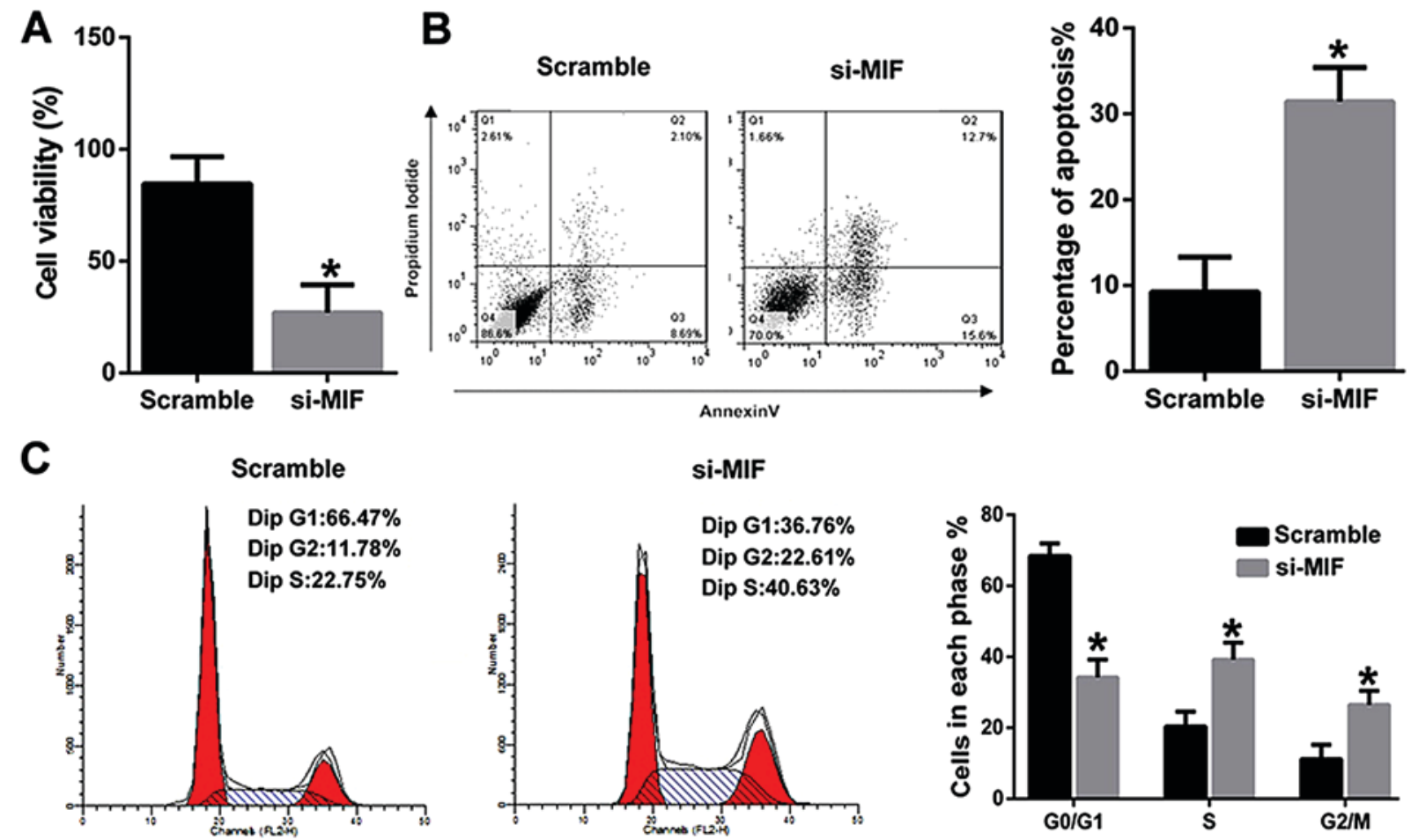

Figure 4. MIF knockdown inhibited the viability and induced the apoptosis of PVM/Ms from young mice (A) MIF knockdown significantly inhibited the viability of PVM/Ms as compared to the control. (B) MIF knockdown significantly induced the apoptosis of PVM/Ms as compared to the control. (C) The cell population in G0/G1 phase of PVM/Ms with MIF knockdown was significantly decreased as compared to the control; whereas the cell population in S and G2/M phases of PVM/Ms with MIF knockdown was significantly increased as compared to the control. MIF silencing in vitro was performed on passage-3 PVM/Ms seeded in 24-well plates as described previously (12). 4 days after the transfection of si-MIF or scrambled siRNA, the transfection efficiency was determined by western blotting and cells were used for cell viability and apoptosis assay. " $\mathrm{P}<0.5$ vs. the Scramble group. PVM/Ms, perivascular-resident macrophage-like melanocytes; MIF, migration inhibitory factor.

function. In our study, we found the reduction of MIF in aged mice. Additionally, MIF knockdown in young mice not only leads to hearing loss, but also inhibited the viability and induced the apoptosis of PVM/Ms. Besides, the cell population in G0/G1 phase of PVM/Ms with MIF knockdown was significantly decreased, and the cell population in $\mathrm{S}$ and $\mathrm{G} 2 / \mathrm{M}$ phases of PVM/Ms with MIF knockdown was significantly increased as compared to the control. The progression of cell cycle was arrested at $\mathrm{S}$ and $\mathrm{G} 2 / \mathrm{M}$ phases as compared to the control. Dong et al indicated that genes involved in apoptosis were associated with human presbycusis, and provided important evidence to support the suggestion that apoptosis was involved in the pathogenesis of human presbycusis (23). MIF was reported to regulate cell proliferation, survival and apoptosis in many studies (24-26). However, the effects of MIF on the growth of PVM/Ms were not reported previously. In our study, MIF may be identified as a contribute factor to the pathogenesis of presbycusis.

MIF was observed in the spiral ligament, Reissner's membrane, stria vascularis, saccular macula, spiral ganglion cells, and membranous labyrinth, which suggest the important role of MIF in the inner ear of mice (13). Researchers also indicated that MIF-deficient mice had significant loss of cochlear hair cells and prolonged hearing loss after intense noise exposure, indicating that MIF may play an important role in recovery from acoustic trauma and management of MIF may be a novel therapeutic option for noise-induced hearing loss (27). However, the underlying mechanism has not been clear yet, our study may provide a possibility, that MIF may exert its role in hearing function through regulating PVM/Ms growth.

Totally, our results revealed that MIF knockdown in vivo significantly accentuated hearing loss in young mice, and MIF knockdown in vitro significantly inhibited viability and induced apoptosis of PVM/Ms. Our study may provide a potential therapy and prevention method for presbycusis.

\section{Acknowledgements}

This study was supported by grants from the National Natural Science Youth Foundation of China (no. 81500799) and Youth Funds from the First Affiliated Hospital of Zhengzhou University.

\section{References}

1. Heman-Ackah SE, Huang TC and Juhn SK: R442-Antioxidant therapy prevents presbycusis In C57BL6 mice. Otolaryngology Head \& Neck Surgery 139: 2S1, 2008.

2. Gopinath B, Rochtchina E, Wang JJ, Schneider J, Leeder SR and Mitchell P: Prevalence of age-related hearing loss in older adults: Blue mountains study. Arch Intern Med 169: 415-416, 2009.

3. Frisina DR and Frisina RD: Speech recognition in noise and presbycusis: Relations to possible neural mechanisms. Hear Res 106: 95-104, 1997.

4. Gates GA and Mills JH: Presbycusis. Lancet 366: 1111-1120, 2005.

5. Martin del Campo HN, Measor KR and Razak KA: Parvalbumin immunoreactivity in the auditory cortex of a mouse model of presbycusis. Hear Res 294: 31-39, 2012. 
6. Juhn SK, Hunter BA and Odland RM: Blood-labyrinth barrier and fluid dynamics of the inner ear. Int Tinnitus J 7: 72-83, 2001

7. Juhn SK, Rybak LP and Prado S: Nature of blood-labyrinth barrier in experimental conditions. Ann Otol Rhinol Laryngol 90 135-141, 1981.

8. Salt AN, Mleichar I and Thalmann MD: Mechanisms of endocochlear potential generation by stria vascularis. Laryngoscope 97 984-991, 1987.

9. Takeuchi S, Ando M, Sato T and Kakigi A: Three-dimensional and ultrastructural relationships between intermediate cells and capillaries in the gerbil stria vascularis. Hear Res 155: 103-112, 2001.

10. Dai M, Yang Y, Omelchenko I, Nuttall AL, Kachelmeier A, Xiu R and Shi X: Bone marrow cell recruitment mediated by inducible nitric oxide synthase/stromal cell-derived factor-1alpha signaling repairs the acoustically damaged cochlear blood-babyrinth barrier. Am J Pathol 177: 3089-3099, 2010.

11. Shi X: Resident macrophages in the cochlear blood-labyrinth barrier and their renewal via migration of bone-marrow-derived cells. Cell Tissue Res 342: 21-30, 2010.

12. Zhang W, Dai M, Fridberger A, Hassan A, Degagne J, Neng L, Zhang F, He W, Ren T, Trune D, et al: Perivascular-resident macrophage-like melanocytes in the inner ear are essential for the integrity of the intrastrial fluid-blood barrier. Proc Natl Acad Sci USA 109: 10388-10393, 2012.

13. Kariya S, Okano M, Maeda Y, Hirai H, Higaki T, Noyama Y, Haruna T, Nishihira J and Nishizaki K: Role of macrophage migration inhibitory factor in age-related hearing loss. Neuroscience 279: 132-138, 2014.

14. Obikane Y: The role of macrophage migration inhibitory factor (MIF) in cell proliferation signal transmission. Hokkaido Igaku Zasshi 79: 659-666, 2004 (In Japanese).

15. Nishihira J: Macrophage migration inhibitory factor (MIF): Its essential role in the immune system and cell growth. J Interferon Cytokine Res 20: 751-762, 2000.

16. Keithley EM, Canto C, Zheng QY, Fischel-Ghodsian N and Johnson KR: Age-related hearing loss and the ahl locus in mice. Hear Res 188: 21-28, 2004.

17. Hunter KP and Willott JF: Aging and the auditory brainstem response in mice with severe or minimal presbycusis. Hear Res 30: 207-218, 1987.
18. Neng L, Zhang W, Hassan A, Zemla M, Kachelmeier A, Fridberger A, Auer M and Shi X: Isolation and culture of endothelial cells, pericytes and perivascular resident macrophage-like melanocytes from the young mouse ear. Nat Protoc 8: 709-720, 2013.

19. Kaur T, Mukherjea D, Sheehan K, Jajoo S, Rybak LP and Ramkumar V: Short interfering RNA against STAT1 attenuates cisplatin-induced ototoxicity in the rat by suppressing inflammation. Cell Death Dis 2: e180, 2011.

20. Makary CA, Shin J, Kujawa SG, Liberman MC and Merchant SN: Age-related primary cochlear neuronal degeneration in human temporal bones. J Assoc Res Otolaryngol 12: 711-717, 2011.

21. Zhang F, Zhang J, Neng L and Shi X: Characterization and inflammatory response of perivascular-resident macrophage-like melanocytes in the vestibular system. J Assoc Res Otolaryngol 14: 635-643, 2013

22. Neng L, Zhang J, Yang J, Zhang F, Lopez IA, Dong M and Shi X: Structural changes in thestrial blood-labyrinth barrier of aged C57BL/6 mice. Cell Tissue Res 361: 685-696, 2015.

23. Dong Y, Li M, Liu P, Song H, Zhao Y and Shi J: Genes involved in immunity and apoptosis are associated with human presbycusis based on microarray analysis. Acta Oto-laryngol 134: 601-608, 2014.

24. Nguyen MT, Lue H, Kleemann R, Thiele M, Tolle G, Finkelmeier D, Wagner E, Braun A and Bernhagen J: The cytokine macrophage migration inhibitory factor reduces pro-oxidative stress-induced apoptosis. J Immunol 170: 3337-3347, 2003.

25. Baumann R, Casaulta C, Simon D, Conus S, Yousefi S and Simon HU: Macrophage migration inhibitory factor delays apoptosis in neutrophils by inhibiting the mitochondria-dependent death pathway. FASEB J 17: 2221-2230, 2003.

26. Saksida T, Stosic-Grujicic S, Timotijevic G, Sandler S and Stojanovic I: Macrophage migration inhibitory factor deficiency protects pancreatic islets from palmitic acid-induced apoptosis. Immunol Cell Biol 90: 688-698, 2012

27. Kariya S, Okano M, Maeda Y, Hirai H, Higaki T, Noyama Y, Haruna T, Nishihira J and Nishizaki K: Macrophage migration inhibitory factor deficiency causes prolonged hearing loss after acoustic overstimulation. Otol \& Neurot 36: 1103-1108, 2015. 